\title{
Transient weakening of geomagnetic shield probed by GRAPES-3 experiment
}

\author{
P.K. Mohanty ${ }^{* a, b}$, K.P. Arunbabu ${ }^{a, b}$, S.R. Dugad ${ }^{a, b}$, S.K. Gupta ${ }^{a, b}$, B. Hariharan ${ }^{a, b}$, Y. \\ Hayashi $^{a, d}$, P. Jagadeesan ${ }^{a, b}$, A. Jain ${ }^{a, b}$, S. Kawakami ${ }^{a, d}$, H. Kojima ${ }^{a, e}$, S.D. Morris ${ }^{a, b}$, \\ A. Oshima ${ }^{a, f}$, B.S. Rao ${ }^{a, b}$, S. Shibata ${ }^{a, f}$, P. Subramanian ${ }^{a, c}$ \\ ${ }^{a}$ GRAPES-3 Experiment, Cosmic Ray Laboratory, Raj Bhavan, Ooty 643 001, India. \\ ${ }^{b}$ Tata Institute of Fundamental Research, Homi Bhabha Road, Mumbai 400 005, India. \\ ${ }^{c}$ Indian Institute of Science Education and Research, Pune 411021, India \\ ${ }^{d}$ Graduate School of Science, Osaka City University, Osaka 558-8585, Japan \\ ${ }^{e}$ Faculty of Engineering, Aichi Institute of Technology, Toyota City, Aichi 470-0392, Japan \\ ${ }^{f}$ College of Engineering, Chubu University, Kasugai, Aichi 487-8501, Japan \\ E-mail: pkmetifr.res.in
}

The large area $\left(560 \mathrm{~m}^{2}\right)$ GRAPES-3 tracking muon telescope in Ooty, India recorded a 2 hour (h) muon burst on 22 June 2015 starting at 19:00 UT in the midst of a continuing Forbush decrease. The burst occurred following the arrival of a coronal mass ejection containing a $40 \mathrm{nT}$ south-ward interplanetary magnetic field (IMF) that had triggered a G4 class geomagnetic storm. During the 2 hour, the muon telescope recorded an excess of $\sim 10^{6}$ muons with a significance of $54 \sigma$. Simultaneous occurrence of the burst was observed in 9 directions covering 2.3 sr field of view suggesting its origin close to Earth. Trajectory computation of cosmic ray primary protons followed by atmospheric simulation by superimposing the 2 hour IMF variation on geomagnetic field (GMF) could reproduce the observed profiles with a high degree of correlation $(0.89 \pm 0.05)$. The simulations could explain the burst profiles due to lowering of cutoff rigidity $(0.5-0.7 \mathrm{GV})$ for $2 \mathrm{~h}$ which allowed an excess near threshold galactic cosmic rays (GCRs) to enter into the atmosphere. It showed that the near cutoff rigidity GCRs were deflected $210^{\circ}$ in longitude offering a natural explanation of its midnight detection by the GRAPES-3 experiment. This exciting observation has attracted considerable global attention following its publication in Physical Review Letters. Further studies of this event since then have shed more light on this phenomenon which will be presented during the conference.

35th International Cosmic Ray Conference - ICRC2017

10-20 July, 2017

Bexco, Busan, Korea

* Speaker. 


\section{Introduction}

The GRAPES-3 large area $\left(560 \mathrm{~m}^{2}\right)$ tracking muon telescope located at Ooty in India $\left(11.4^{\circ} \mathrm{N}\right.$, $76.7^{\circ} \mathrm{E}$ and $2200 \mathrm{~m}$ altitude) has been operating since 2000 . It measures the galactic cosmic ray (GCR) flux at high geomagnetic cutoff rigidities (14 - $32 \mathrm{GV})$. It comprises 16 individual modules of $35 \mathrm{~m}^{2}$ area each which are based on proportional counters (PRCs). Each PRC is made of steel tubes of $6 \mathrm{~m}$ long and $10 \mathrm{~cm} \times 10 \mathrm{~cm}$ square cross section filled with P-10 gas (90\% $\mathrm{Ar}+10 \%$ $\mathrm{CH}_{4}$ ) which serves as active detection medium. In each module, four layers of total 232 PRCs have been placed in a grid configuration that allows to track and reconstruct the arrival direction of each muon incident at zenith angle up to $60^{\circ}$ covering a field of view of $2.3 \mathrm{sr}$ [1]. Concrete of thickness $550 \mathrm{~g} \mathrm{~cm}^{-2}$ has been placed above the PRC layers that allows penetrating muons of above $1 \mathrm{GeV}$ to be recorded. The reconstructed muons are recorded in $13 \times 13$ (169) directional bins with angular resolution varying from $6^{\circ}$ in vertical to $2^{\circ}$ in the inclined bin at $60^{\circ}$ zenith angle. The muon telescope provides a two dimensional real-time monitor of the muon flux. It records $\sim 5 \times 10^{4}$ muons per second providing high temporal measurement of the GCR modulation by various solar phenomena.

The unique combination of high statistics and high angular resolution measurement of the muon flux has given the GRAPES-3 experiment unprecedented sensitivity to study space weather, driven by transient solar events such as flares and coronal mass ejections (CMEs) [1, 2, 3, 4, 5, 6]. Space weather is a major concern since it can cause significant disruptions in our immediate space environment, affecting a whole host of technologies such as the global positioning system, satellite operations, communication, aviation and the electrical power grids that we routinely rely on [7].

\section{Observation of muon burst on 22 June 2015 by GRAPES-3}

The GRAPES-3 muon telescope recorded an unusual increase in the muon rate on 22 June 2015 which started at 19:00 UT and lasted for two hours as can be seen from Fig.1. The effect subsequently referred as the burst occurred in the midst of a continuing Forbush Decrease (FD) that started around 20:00 UT on 21 June 2015. The FD reached a minimum change of $-2.5 \%$ in the muon intensity around 12:00 UT on 23 June 2015. However, it took about eight days for the recovery.

The burst was investigated in connection with CME as discussed below. During the period 18 - 25 June, 2015, a series of solar flares and CMEs were erupted from an active sunspot region NOAA AR2371. The WIND spacecraft measurements at L1 location time shifted to Earth's bow shock nose showed that three CMEs arrived Earth in succession on 21 June at 16:45 UT, 22 June at 5:45 UT and 22 June at 18:40 UT [9]. Their arrivals are marked by jumps in solar wind velocity $\left(\mathrm{V}_{\mathrm{sw}}\right)$ and total IMF $(|\mathrm{B}|)$ as shown in Fig.2a and Fig.2b respectively. When the third CME struck at 18:40 UT, the $\mathrm{V}_{\mathrm{sw}}$ increased from $440 \mathrm{~km} / \mathrm{s}$ to $700 \mathrm{~km} / \mathrm{s}$ and the IMF $|\mathrm{B}|$ increased from $10 \mathrm{nT}$ to $44 \mathrm{nT}$. The $\mathrm{B}_{z}$ (the component of IMF perpendicular to ecliptic plane) changed to $-40 \mathrm{nT}$ implying a strong south orientation (Fig.2c) of the IMF. However, the first and second CME did not show any significant variation in the $\mathrm{B}_{z}$. The third CME was associated with the eruption of a double peaked M2-class solar flare which had appeared 40 hours earlier in the SOHO/LASCO C2 images at 02:36 UT on 21 June 2015. 


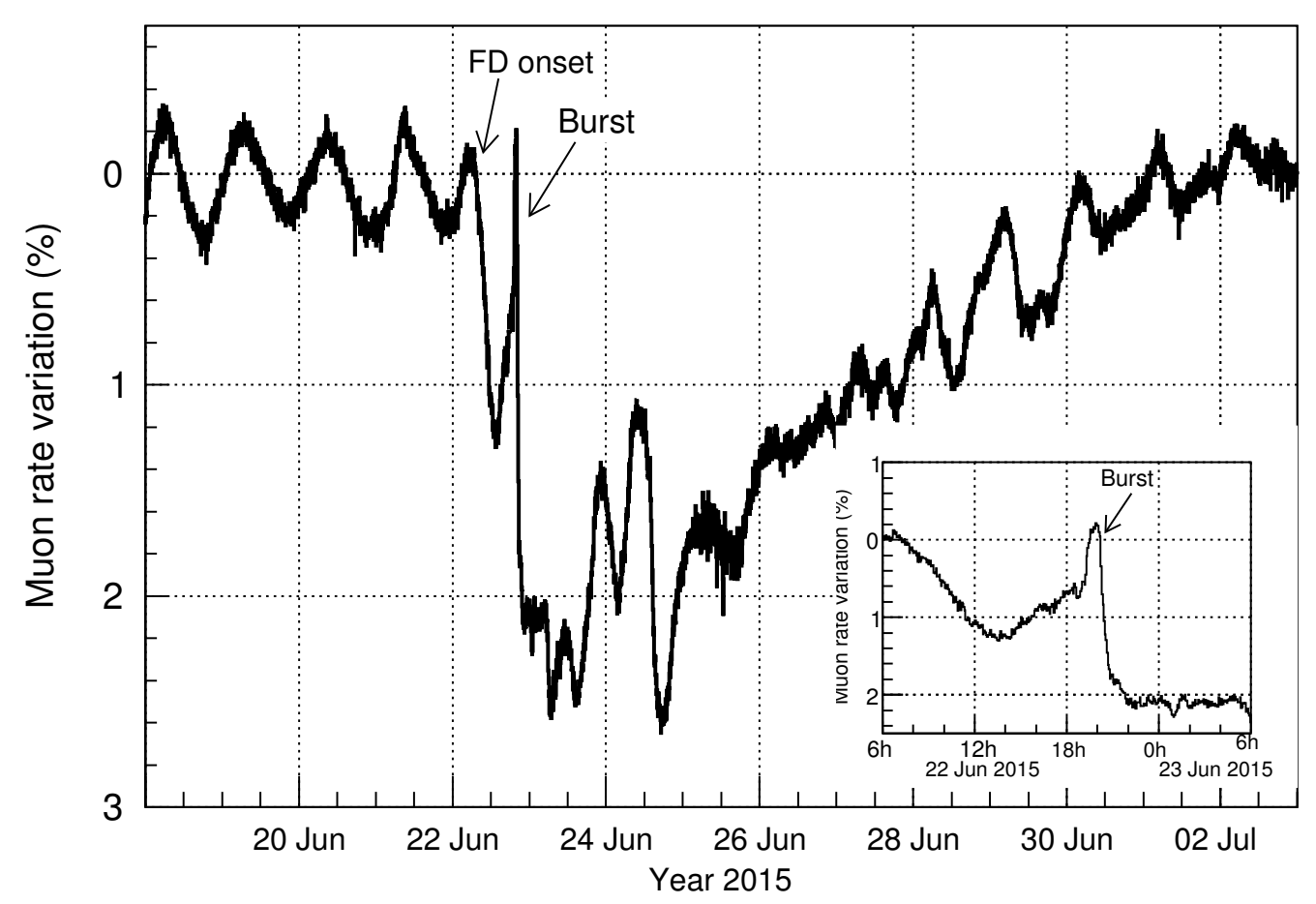

Figure 1: The muon burst in the middle of a continuing FD observed on 22 June 2015 by GRAPES-3 muon telescope. The mini plot shows the zoomed view of the burst.

To separate the burst signal from the muon rate data modulated by solar diurnal anisotropy (SDA) variation at a frequency of 1 cycle per day (cpd) and by two higher harmonics to lesser extent and the slow FD variations as shown in Fig.1, a first Fourier transform (FFT) based analysis was carried out. The muon rates were first corrected for the instrumental and atmospheric pressure effects [8]. To implement FFT, the muon rates measured every four min for $2^{13}=8192$ intervals spanning 23 days from 12 June 2015 18:28 UT to 4 July 2015 12:36 UT were used. The SDA contributions were removed using a filter on the FFT spectrum by rejecting frequencies from 0.5 to $3.5 \mathrm{cpd}$. The inverse FFT of the filtered spectrum is shown in Fig.2d. As it could be seen, a FD was in progress $4.5 \mathrm{~h}$ after the arrival of the first CME on 21 June at 16:45 UT. In the decrease phase of the FD, a $2 \mathrm{~h}$ muon burst (19:00- 21:00 UT) correlated with $\mathrm{B}_{z}$ is clearly seen from Fig.2c, and Fig. $2 \mathrm{~d}$ respectively, as indicated by the arrows.

To remove both SDA and slow FD contributions, another filter was used to eliminate all the frequency components up to $3.5 \mathrm{cpd}$. The filtered muon rate and $-\mathrm{B}_{z}$ data at 4 min resolution are shown in Fig.3. The maximum correlation $(R=-0.94)$ was obtained when $B_{Z}$ was delayed by 32 min with respect to the muon time. This indicated that the muon burst measured on the ground has a strong connection with the $\mathrm{B}_{z}$. During the $2 \mathrm{~h}$ (19:00 UT - 21:00UT), $2 \times 10^{5}$ excess muons were recorded on a background of $2.9 \times 10^{8}$ muons with an estimated significance of $54 \sigma$.

The burst measured by the muon telescope in nine independent directions namely N, NW, NE, 


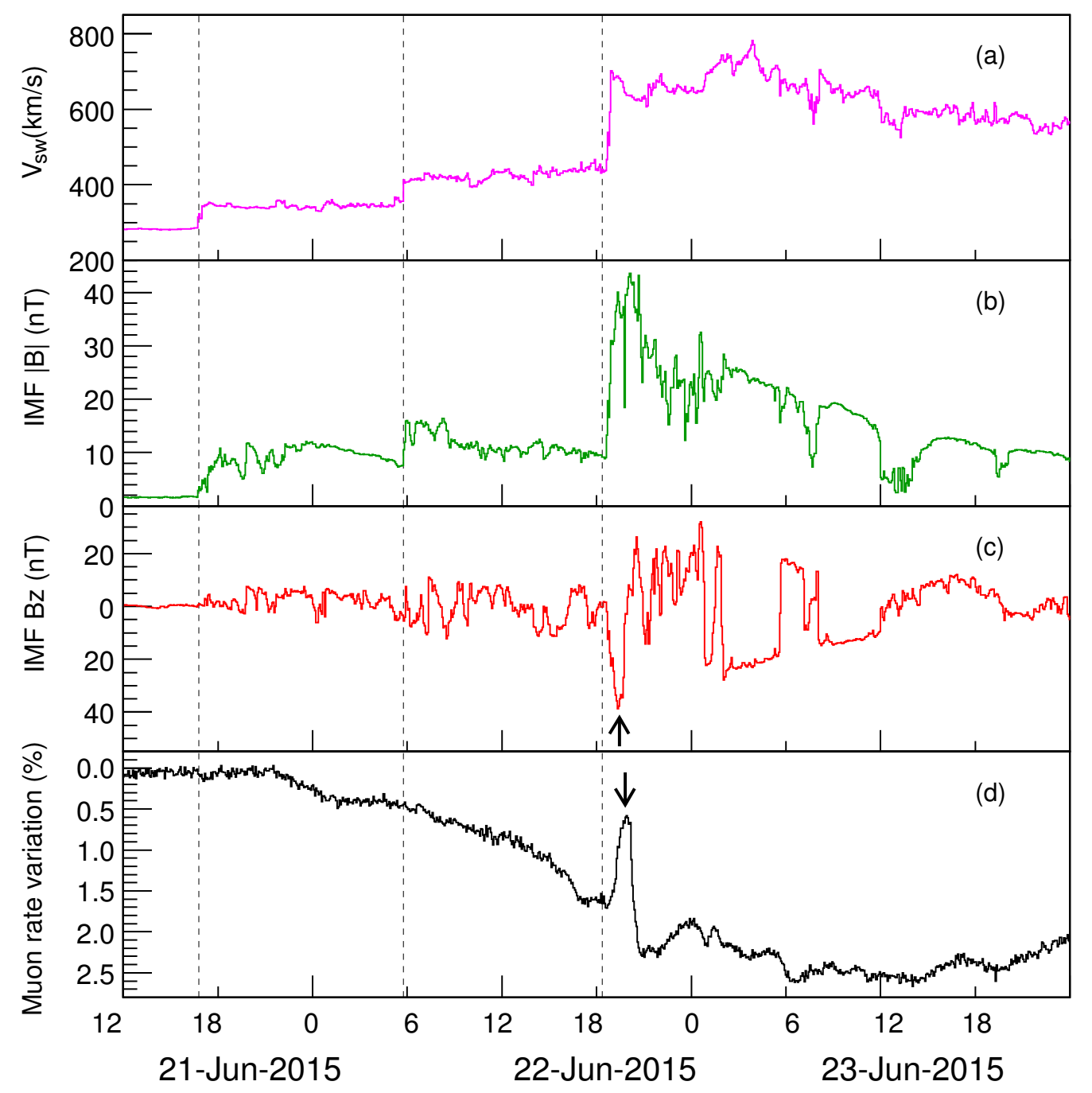

Figure 2: Top 3 panels show solar wind and IMF data from WIND spacecraft time shifted to the bow shock nose: (a) $\mathrm{V}_{\mathrm{SW}}$, (b) $|\mathrm{B}|$, (c) $\mathrm{B}_{\mathrm{Z}}$, (d) GRAPES-3 muon rate. Vertical dashed lines indicate CME arrival times (UT).

W, V, E, SW, S, SE covering its field of view with nearly equal solid angles [1] was obtained. The SDA and FD variations were removed using FFT filter as discussed earlier and the burst profiles are shown in Fig.4. The cutoff rigidity in the nine directions varies from 15 to $24 \mathrm{GV}$. The burst amplitudes are higher in north directions $(\mathrm{N}=1.8 \%)$ and lower in south directions $(\mathrm{SW}=0.8 \%)$. Each direction showed a simultaneous increase in the muon rate from 19:00 UT and reached a maximum at 20:00 UT. For an interplanetary phenomenon, the expected time offset between V, and directions NE, E, SE is -100 min. Similarly, the offset between V, and NW, W, SW is 100 min based on the $25^{\circ}$ angle between them. However, the observed offsets between $\mathrm{V}$, and NE, E, 


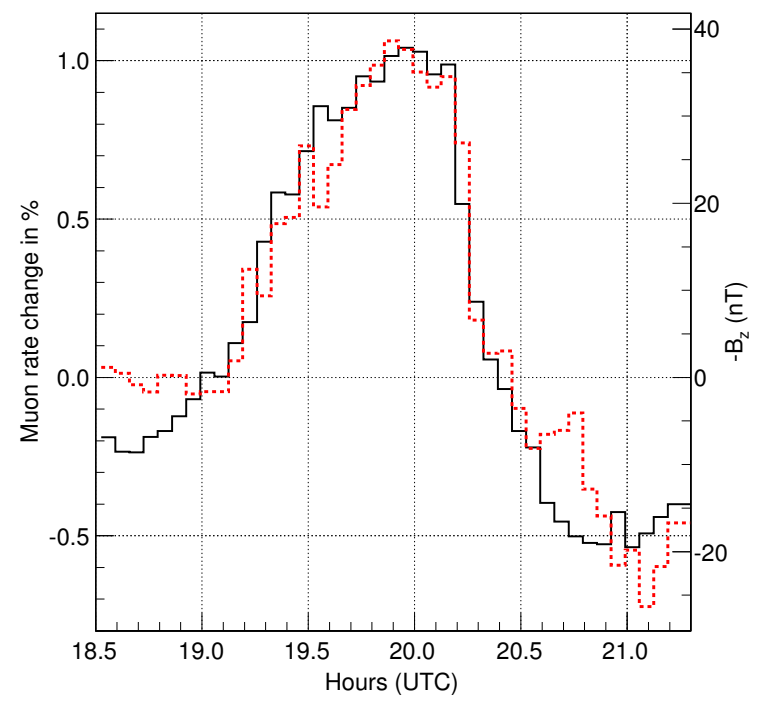

Figure 3: Correlation between muon rate (solid line) and IMF $B_{Z}$ (broken line) during the 22 June 2015 burst event. Correlation coefficient $\mathrm{R}=-0.94$

SE, NW, W, SW, measured via a cross correlation yielded a mean of $-3 \pm 4$ min, consistent with a value of zero within the 4 min time resolution. As expected, the time offsets between $\mathrm{V}$, and $\mathrm{N}, \mathrm{S}$ were also zero. Thus, the near simultaneity of the burst in all directions strongly suggested that it originated very close to Earth.

\section{Simulation of 22 June 2015 burst}

Since the burst occurrence was simultaneous in all directions, we started with a hypothesis that a lowering of cutoff rigidity $R_{c}$ could have allowed more low energy cosmic rays to enter Earth leading to the enhancement in the flux. To test this hypothesis, we recalculated the $R_{c}$ for a model geomagnetic field (GMF) perturbed by the external IMF during the the $2 \mathrm{~h}$ burst. $\mathrm{R}_{\mathrm{c}}$ values were calculated with fine spatial resolution by dividing the muon telescope field of view into $360^{\circ} \times 60^{\circ}$ grid, each $1^{\circ}$ in azimuth and zenith directions. The GMF was modeled by vectorially adding the GMF components calculated from the IGRF-11 model [10] to the respective IMF components $\left(\mathrm{B}_{x}\right.$, $\mathrm{B}_{y}, \mathrm{~B}_{z}$ averaged every $4 \mathrm{~min}$ ). Before the addition, the IMF components were converted from GSE to geocentric coordinates. Cutoff rigidity for a given direction was obtained by computing trajectory of a antiproton in the model field up to $25 \mathrm{R}_{\mathrm{E}}$ [11]. The computation was performed in a range of rigidity values starting $2 \mathrm{GV}$ above to $2 \mathrm{GV}$ below from an expected cutoff rigidity for each direction at interval of $0.01 \mathrm{GV}$.

The production of muons in the atmosphere due to interaction of cosmic rays above $\mathrm{R}_{c}$ was simulated by CORSIKA [12]. The simulated muons satisfying the trigger requirements of the muon telescope were binned into the nine directions mentioned above. The difference between the muon rates obtained from the simulation without and with the IMF were calculated for every $4 \mathrm{~min}$. The simulated amplitudes were significantly smaller $(\sim 0.05 \%)$ than the measured $(\sim 1.0 \%)$ ones. 


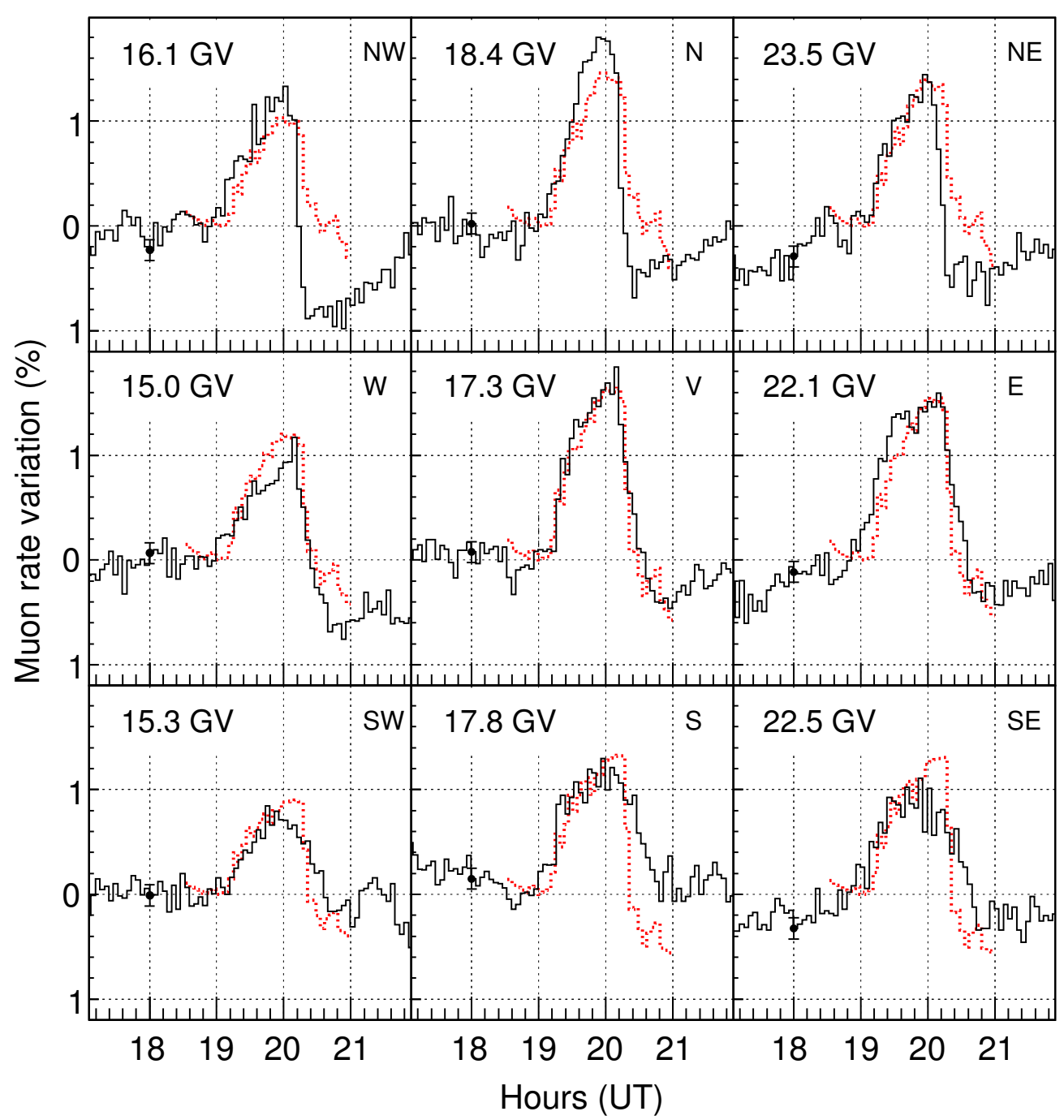

Figure 4: Muon rate variation in nine directions observed by GRAPES-3 on 22 June 2015 are shown by the solid line. Simulation results obtained by scaling the IMF components 17 times are shown by the broken line. Cutoff rigidities (GV) and error bars are shown for each direction.

These simulations were repeated after enhancing the IMF by a factor $2<\mathrm{f}<20$ and for scale of 17, the simulated amplitudes gave good agreement.

The nine simulated profiles are shown in Fig. 4 by broken lines. Very high correlations $(-0.89 \pm$ 0.05 ) between the measured, and simulated profiles are seen in all nine cases. The reduction in $\mathrm{R}_{c}$ varied from 0.5 in the south to $0.7 \mathrm{GV}$ in the north. It is remarkable that a simple model with a common compression factor $\mathrm{f}=17\left(\mathrm{~B}_{z}=-680 \mathrm{nT}\right)$ reproduced the amplitude, and the shapes of all nine profiles quite well.

Cosmic rays near the cutoff rigidity experience a large deflection in the GMF. To estimate this 
"deflection" asymptotic directions were calculated for $5 \times 10^{4}$ protons. Protons of rigidities from $\mathrm{R}_{c} i$ to $\mathrm{R}_{c} i \pm \Delta \mathrm{R}_{c i} \mathrm{GV}$ for $\mathrm{i}=1,9$ directions were simulated. Here $\Delta \mathrm{R}_{c i}$, were the changes in the respective cutoff rigidities $(0.5-0.7 \mathrm{GV})$. Trajectories for the most probable rigidity for the nine directions, viewed from the equator are shown in Fig.5. These trajectories are bending $195^{\circ}-230^{\circ}$; thus, the asymptotic directions lie in the opposite hemisphere. Thus, the cosmic rays producing the muon burst detected on the night side were deflected $\sim 210^{\circ}$ from the day side.

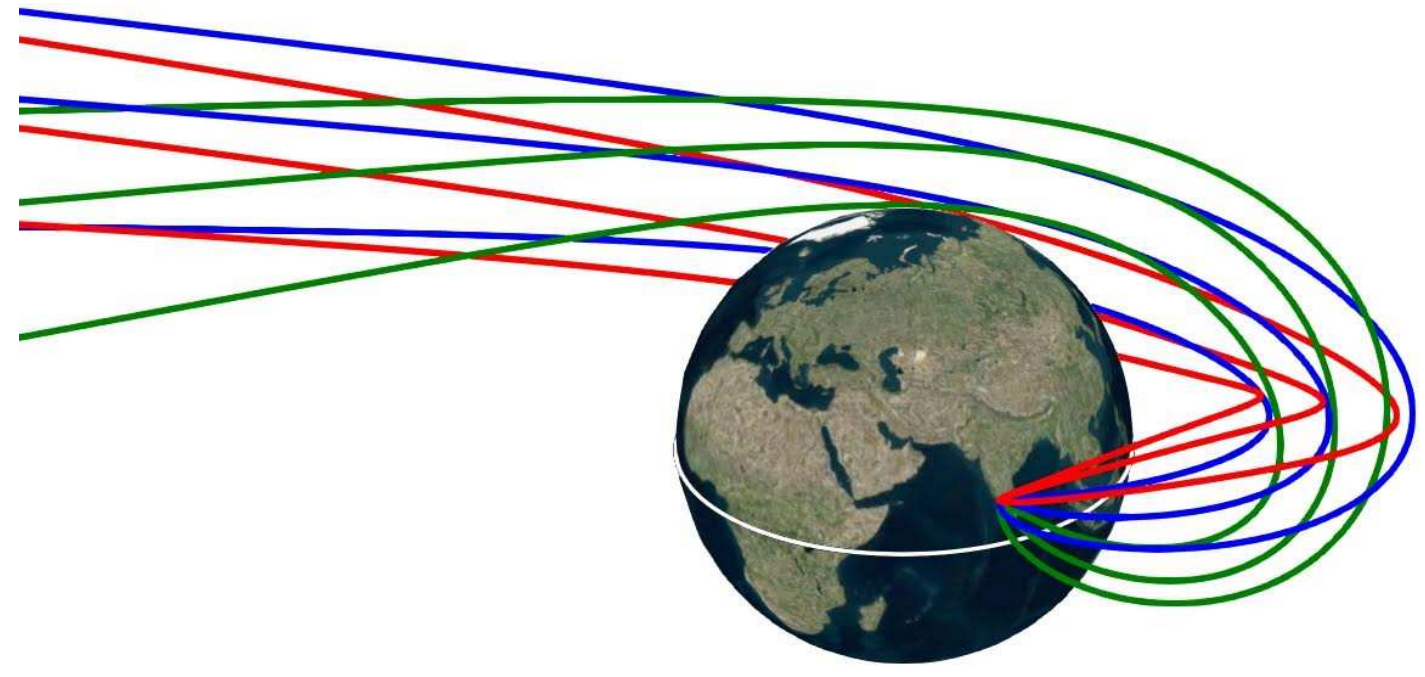

Figure 5: Cosmic ray trajectories near the cutoff rigidity responsible for the burst viewed from the equator. NW, N, NE shown in blue, W, V, E in green, SW, S, SE in red.

\section{Conclusions}

The GRAPES-3 muon telescope in Ooty, India detected a $2 \mathrm{~h}$ burst of GCRs starting 22 June 2015 19:00 UT that was strongly correlated with a $40 \mathrm{nT}$ surge in the IMF. Monte Carlo simulations showed compression of IMF from $40 \mathrm{nT}$ to $680 \mathrm{nT}$ and subsequent reconnection with the GMF leading to lower cutoff rigidities may have generated this burst. The $\mathrm{B}_{\mathrm{z}}$ could have been enhanced by the compression of the magnetosphere. The WIND measurement shows that the bow shock nose was compressed from 11.4 to $4.6 \mathrm{R}_{\mathrm{E}}$. The simulation showed the near cutoff rigidity particles deflected $\sim 210^{\circ}$ enabling the detection by GRAPES-3 at night side. Our examination of the worldwide neutron monitor data publicly available showed that Almaty and Nor-Amberd stations located on the night side recorded increased rates coincident with GRAPES-3 [13]. The burst showed a 32 min delay relative to the IMF. Although, a clear understanding for the delay yet to emerge, but it may help in space weather forecast.

\section{Acknowledgement}

We thank D.B. Arjunan, G.P. Francis, V. Jeyakumar, S. Kingston, K. Manjunath, S. Murugapandian, S. Pandurangan, B. Rajesh, K. Ramadass, V. Santoshkumar, M.S. Shareef, C. Shobana, R. 
Sureshkumar and other colleagues for their help in running and maintanence of the GRAPES-3 experiment.

\section{References}

[1] T. Nonaka et al., Phys. Rev. D 74 (2006) 052003.

[2] K. P. Arunbabu et al., Astron. Astrophys., 555 (2013) A139.

[3] K. P. Arunbabu et al., Astron. Astrophys. 580 (2015) A41.

[4] H. Kojima et al., Astroparticle Phys. 62 (2015) 21.

[5] H. Kojima et al., PRD 62 (2015) 121303(R).

[6] P.K. Mohanty et al., PRL 117 (2016) 171101.

[7] Ferguson et al., IEEE TRANSACTIONS ON PLASMA SCIENCE 43 (2015) 3086.

[8] P.K. Mohanty et al., Astropart. Phys., 79 (2016) 2330.

[9] http://omniweb.gsfc.nasa.gov/form/omni_min.html.

[10] C. Finlay et al., Geophys. J. Int. 183 (2010) 1216.

[11] D. F. Smart and M. A. Shea, Adv. Space Res. 36 (2005) 2012.

[12] https://www.ikp.kit.edu/corsika/

[13] http://www.nmdb.eu 